\title{
LIE NILPOTENCY INDICES OF MODULAR GROUP ALGEBRAS
}

\author{
V. BOVDI AND J. B. SRIVASTAVA
}

\begin{abstract}
Let $K$ be a field of positive characteristic $p$ and $K G$ the group algebra of a group $G$. It is known that if $K G$ is Lie nilpotent then its upper (or lower) Lie nilpotency index is at most $\left|G^{\prime}\right|+1$, where $\left|G^{\prime}\right|$ is the order of the commutator subgroup. The class of groups $G$ for which these indices are maximal or almost maximal have already been determined. Here we determine $G$ for which upper (or lower) Lie nilpotency index is the next highest possible.
\end{abstract}

\section{INTRODUCTION AND RESULTS}

Let $R$ be an associative algebra with identity. The algebra $R$ can be regarded as a Lie algebra, called the associated Lie algebra of $R$, via the Lie commutator $[x, y]=x y-y x$, for every $x, y \in R$. Set $\left[x_{1}, \ldots, x_{n}\right]=\left[\left[x_{1}, \ldots, x_{n-1}\right], x_{n}\right]$, where $x_{1}, \ldots, x_{n} \in R$. The $n$-th lower Lie power $R^{[n]}$ of $R$ is the associative ideal generated by all the Lie commutators $\left[x_{1}, \ldots, x_{n}\right]$, where $R^{[1]}=R$ and $x_{1}, \ldots, x_{n} \in R$. By induction, we define the $n$-th upper Lie power $R^{(n)}$ of $R$ as the associative ideal generated by all the Lie commutators $[x, y]$, where $x \in R^{(n-1)}, y \in R$ and $R^{(1)}=R$.

An algebra $R$ is called lower Lie nilpotent (respectively upper Lie nilpotent) if there exists $m$ such that $R^{[m]}=0 \quad\left(R^{(m)}=0\right)$. The minimal integers $m, n$ such that $R^{[m]}=0$ and $R^{(n)}=0$ are called the lower Lie nilpotency index and the upper Lie nilpotency index of $R$ and these are denoted by $t_{L}(R)$ and $t^{L}(R)$, respectively.

We would like to mention that N. Gupta and F. Levin 9 have given an example of algebra $R$ for which $t_{L}(R)=3$ but $R$ is not upper Lie nilpotent. Moreover in that paper, it was shown that if $R$ is a lower Lie nilpotent ring of class $n$, then the unit group $U(R)$ is nilpotent of class at most $n$.

An algebra $R$ is called Lie hypercentral if for each sequence $\left\{a_{i}\right\}$ of elements of $R$ there exists some $n$ such that $\left[a_{1}, \ldots, a_{n}\right]=0$.

Let $U(K G)$ be the group of units of a group algebra $K G$. For the noncommutative modular group algebra $K G$ the following Theorem due to A.A. Bovdi, I.I. Khripta, I.B.S. Passi, D.S. Passman and etc. (see [2, 11, 13]) is well known: The following statements are equivalent: (a) $K G$ is lower Lie nilpotent (b) $K G$ is Lie hypercentral; (c) $K G$ is upper Lie nilpotent (d) $U(K G)$ is nilpotent; (e) $\operatorname{char}(K)=p>0, G$ is nilpotent and its commutator subgroup $G^{\prime}$ is a finite $p$ group.

It is well known (see [17]) that, if $K G$ is Lie nilpotent, then

$$
t_{L}(K G) \leq t^{L}(K G) \leq\left|G^{\prime}\right|+1 .
$$

1991 Mathematics Subject Classification. 16S34, 17B30.

Key words and phrases. Group algebras, Lie nilpotency index, Lie dimension subgroups.

The research was supported by OTKA grants No. T 43034, No. K 61007. 
According to [1], if $\operatorname{char}(K)>3$, then $t_{L}(K G)=t^{L}(K G)$. But the question of when $t_{L}(K G)=t^{L}(K G)$ for $\operatorname{char}(K)=2,3$ is in general still open.

A.Shalev in [16] began the study of the question when the Lie nilpotent group algebra $K G$ has the maximal lower Lie nilpotency index. In 6, 16, was given the complete description of such Lie nilpotent group algebras. In [4, 5] we obtained the full description of the Lie nilpotent group algebras $K G$ with upper/lower almost maximal Lie nilpotent indices.

Our main results in this paper are the following theorems.

Theorem 1. Let $K G$ be a Lie nilpotent group algebra over a field $K$ of positive characteristic $p$. Then $t^{L}(K G)=\left|G^{\prime}\right|-4 p+5$ if and only if one of the following conditions holds:

(i) $p=2, \quad c l(G)=2$ and $G^{\prime} \cong C_{2} \times C_{2} \times C_{2}$;

(ii) $p=5, \quad c l(G)=2$ and $G^{\prime} \cong C_{5} \times C_{5}$.

Moreover, $t_{L}(K G)=t^{L}(K G)$.

Theorem 2. Let $K G$ be a Lie nilpotent group algebra over a field $K$ of positive characteristic $p$. Then $t^{L}(K G)=\left|G^{\prime}\right|-3 p+4$ if and only if one of the following conditions holds:

(i) $p=2, \quad \operatorname{cl}(G)=3, \quad G^{\prime} \cong C_{2} \times C_{2} \times C_{2}$ and $\gamma_{3}(G)$ is cyclic;

(ii) $p=2, \quad G^{\prime} \cong C_{4} \times C_{2}$ and $\gamma_{2}(G)^{2} \subseteq \gamma_{3}(G)$;

(iii) $p=5, \quad c l(G)=3$ and $G^{\prime} \cong C_{5} \times C_{5}$.

Moreover, $t_{L}(K G)=t^{L}(K G)$.

Theorem 3. Let $K G$ be a Lie nilpotent group algebra over a field $K$ of positive characteristic $p$. Then $t^{L}(K G)=\left|G^{\prime}\right|-2 p+3$ if and only if one of the following conditions holds:

(i) $p=2, \quad \operatorname{cl}(G)=3, \quad G^{\prime} \cong C_{2} \times C_{2} \times C_{2} \quad$ and $\quad \gamma_{3}(G) \cong C_{2} \times C_{2}$;

(ii) $p=2, \quad G^{\prime}=\langle a\rangle \times\langle b\rangle \cong C_{4} \times C_{2}$ and $\gamma_{3}(G)$ is one of the following groups: $\langle b\rangle,\left\langle a^{2}\right\rangle \times\langle b\rangle,\left\langle a^{2} b\right\rangle$;

(iii) $p=3, \quad \operatorname{cl}(G)=3$ and $G^{\prime} \cong C_{3} \times C_{3}$.

Moreover, $t_{L}(K G)=t^{L}(K G)$.

Theorem 4. Let $K G$ be a Lie nilpotent group algebra over a field $K$ of positive characteristic $p$. Then

(i) $t_{L}(K G)=\left|G^{\prime}\right|-4 p+5$ if and only if $G$ and $K$ satisfies one of the conditions (i)-(ii) of Theorem 1 .

(ii) $t_{L}(K G)=\left|G^{\prime}\right|-3 p+4$ if and only if $G$ and $K$ satisfies one of the conditions (i)-(iii) of Theorem Q

(iii) $t_{L}(K G)=\left|G^{\prime}\right|-2 p+3$ if and only if $G$ and $K$ satisfies one of the conditions (i)-(iii) of Theorem 3 ,

Finally, by Xiankun Du's and I. Khripta's Theorems ([8, 11]) we get

Corollary 1. Let $K G$ be the group algebra of a finite p-group $G$ over a field $K$ of $\operatorname{char}(K)=p>0$ and $U(K G)$ its group of units. Then the nilpotency class of $U(K G)$ is equal to

(i) $\quad\left|G^{\prime}\right|-4 p+4$ if and only if $G$ and $K$ satisfies one of the conditions (i)-(ii) of Theorem 1 .

(ii) $\left|G^{\prime}\right|-3 p+3$ if and only if $G$ and $K$ satisfies one of the conditions

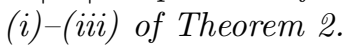


(iii) $\left|G^{\prime}\right|-2 p+2$ if and only if $G$ and $K$ satisfies one of the conditions (i)-(iii) of Theorem 3 .

\section{Preliminaries}

We use the standard notation: $C_{n}$ is the cyclic group of order $n ; \quad \zeta(G)$ is the center of a group $G$ and $(g, h)=g^{-1} h^{-1} g h(g, h \in G) ; \gamma_{i}(G)$ is the $i$-th term of the lower central series of $G$, i.e.

$$
\gamma_{1}(G)=G, \quad \gamma_{i+1}(G)=\left(\gamma_{i}(G), G\right) \quad(i \geq 1) .
$$

Let $K$ be a field of positive characteristic $p, \quad G$ a group and put

$$
\mathfrak{D}_{(m)}(G)=G \cap\left(1+K G^{(m)}\right), \quad(m \geq 1) .
$$

The subgroup $\mathfrak{D}_{(m)}(G)$ is called the $m$-th Lie dimension subgroup of $K G$ and by Theorem 2.8 ([12, p.48) we have:

$$
\mathfrak{D}_{(m+1)}(G)=\left\{\begin{array}{lll}
G & \text { if } & m=0 ; \\
G^{\prime} & \text { if } & m=1 ; \\
\left(\mathfrak{D}_{(m)}(G), G\right)\left(\mathfrak{D}_{\left(\left\lceil\frac{m}{p}\right\rceil+1\right)}(G)\right)^{p} & \text { if } & m \geq 2,
\end{array}\right.
$$

where $\left\lceil\frac{m}{p}\right\rceil$ is the upper integer part of $\frac{m}{p}$.

By [12] (see p.46) there exists an explicit expression for $\mathfrak{D}_{(m+1)}(G)$ :

$$
\mathfrak{D}_{(m+1)}(G)=\prod_{(j-1) p^{i} \geq m} \gamma_{j}(G)^{p^{i}} .
$$

Put $p^{d_{(k)}}=\left[\mathfrak{D}_{(k)}(G): \mathfrak{D}_{(k+1)}(G)\right]$, where $k \geq 1$. If $K G$ is Lie nilpotent, such that $\left|\gamma_{2}(G)\right|=p^{n}$, then according to Jennings' theory [14] for the Lie dimension subgroups, we get

$$
t^{L}(K G)=2+(p-1) \sum_{m \geq 1} m d_{(m+1)}
$$

and it is easy to check that

$$
\sum_{m \geq 2} d_{(m)}=n
$$

For $x, y, z \in G$ we shall use the following well known formula

$$
(x \cdot y, z)=(x, z)(x, z, y)(y, z) .
$$

We begin with the following results by A. Shalev (see Corollary 4.5 and Corollary 4.6 of [15] and Theorem 3.9 of [16]):

Lemma 1. Let $K$ be a field with $\operatorname{char}(K)=p>0$ and $G$ a nilpotent group such that $\left|G^{\prime}\right|=p^{n}$ and $\exp \left(G^{\prime}\right)=p^{l}$.

(i) If $d_{(m+1)}=0$ and $m$ is a power of $p$, then $\mathfrak{D}_{(m+1)}(G)=\langle 1\rangle$.

(ii) If $d_{(m+1)}=0$ and $p^{l-1}$ divides $m$, then $\mathfrak{D}_{(m+1)}(G)=\langle 1\rangle$.

(iii) If $p \geq 5$ and $t_{L}(K G)<p^{n}+1$ then $t_{L}(K G) \leq p^{n-1}+2 p-1$.

(iv) If $d_{(l+1)}=0$ for some $l<p m$ then $d_{(p m+1)} \leq d_{(m+1)}$.

(v) If $d_{(m+1)}=0$ then $d_{(l+1)}=0$ for all $l \geq m$ such that $\nu_{p^{\prime}}(l) \geq \nu_{p^{\prime}}(m)$, where $\nu_{p^{\prime}}(x)$ is the maximal divisor of $x$ which relative prime to $p$.

Fist of all we proof the following: 
Lemma 2. Let $K$ be a field with $\operatorname{char}(K)=p>0$ and $G$ a nilpotent group such that $\left|G^{\prime}\right|=p^{n}$. Then $t^{L}(K G)=\left|G^{\prime}\right|-4 p+5$ if and only if one of the following condition holds:

(i) $p=2, \quad n=3 \quad$ and $\quad d_{(2)}=3$;

(ii) $p=3, \quad n=3 \quad$ and $\quad d_{(2)}=d_{(4)}=d_{(6)}=1$;

(iii) $p=5, \quad n=2 \quad$ and $\quad d_{(2)}=2$.

Proof. Let $t^{L}(K G)=\left|G^{\prime}\right|-4 p+5$, where $p=\operatorname{char}(K)$. If either $n=1$ or $p=n=2$, then according to Theorem 1 of [6], and to Corollary 1 of [5], we get $t^{L}(K G) \geq\left|G^{\prime}\right|$. So in the sequel we assume that either $p=2$ and $n \geq 3$ or $n \geq 2$.

Let $p=2, n=3$ and suppose that $d_{(2)} \leq 2$. By (2) we have that $t^{L}(K G) \geq 6>$ 5 , so only possible case is $d_{(2)}=3$.

Let $p=2, n=4$ and consider the following cases: $d_{(2)}, d_{(3)} \in\{1,2,3\}$. By (21), (3) and (i) of Lemma 1 it is easy to compute that one possible solution is: $d_{(2)}=d_{(3)}=1$ and $d_{(5)}=2$ which in contradictions with (iv) of Lemma 1

Now, let $p=2$ and $n \geq 5$. We prove that $d_{\left(p^{i}+1\right)}>0$ for $\quad 0 \leq i \leq n-2$.

Suppose that $d_{\left(2^{n-2}+1\right)}=0$ and $d_{\left(2^{n-3}+1\right)} \neq 0$. By (i) of Lemma 1 we have that $\mathfrak{D}_{\left(2^{n-2}+1\right)}(G)=\langle 1\rangle$ and $d_{(r)}=0$ for every $r \geq 2^{n-2}+1$. Moreover, if $d_{(q+1)} \neq 0$, then $q<2^{n-2}$. According to (3) it follows that

$$
\begin{aligned}
t^{L}(K G) & =2+\sum_{i=0}^{n-3} 2^{i}+\sum_{i=0}^{n-3} 2^{i}\left(d_{\left(2^{i}+1\right)}-1\right)+\sum_{q \neq 2^{i}} q d_{(q+1)} \\
& <1+\left(1+\sum_{i=0}^{n-3}\left(d_{\left(2^{i}+1\right)}-1\right)+\sum_{q \neq 2^{i}} d_{(q+1)}\right) \cdot 2^{n-2} \\
& =1+(1+n-(n-2)) \cdot 2^{n-2}<2^{n}-3
\end{aligned}
$$

which is contradicts to $t^{L}(K G)=\left|G^{\prime}\right|-3$.

Therefore $d_{\left(p^{i}+1\right)}>0$ for $0 \leq i \leq n-2$ and by (3i) and (4) there exists $\alpha \geq 2$ such that $d_{(\alpha+1)}=1$, and

$$
t^{L}(K G)=2+\sum_{i=0}^{n-2} 2^{i}+\alpha d_{(\alpha+1)}=1+2^{n-1}+\alpha .
$$

Since $t^{L}(K G)=\left|G^{\prime}\right|-3$, it must be $\alpha=2^{n-1}-4$. Put $m=2^{n-3}-1$ and $l=4 m$. Since $\nu_{2^{\prime}}(l)=\nu_{2^{\prime}}(m)$ and $d_{\left(\left(2^{n-3}-1\right)+1\right)}=0$, by (v) of Lemma 1 we get $d_{\left(\left(2^{n}-4\right)+1\right)}=0$, a contradiction.

Let now $p=3$ and $n \geq 4$. Using the same arguments as in the previous case, we get $d_{\left(3^{i}+1\right)}=d_{\left(\left(3^{n-1}-4\right)+1\right)}=1$ for $0 \leq i \leq n-2$. Since $\exp \left(G^{\prime}\right) \leq 3^{n-1}$ and $3^{n-2} \mid 2 \cdot 3^{n-2}$, by (ii) of Lemma 1 it yields that $\mathfrak{D}_{\left(2 \cdot 3^{n-2}+1\right)}(G)=\langle 1\rangle$, so $d_{\left(\left(3^{n-1}-4\right)+1\right)}=0$, a contradiction.

If $n=2$, then by (3) we obtain that $t^{L}(K G) \geq 6>2$.

Let $p=n=3$. According to the case when $p=2$ and $n=4$, it is easy to check that $d_{(2)}=d_{(4)}=d_{(6)}=1$ and the proof for $p=3$ is complete.

Let $p=5$. The inequality $t^{L}(K G)=5^{n}-15 \leq 5^{n-1}+9$ is verified for $n=2$. Suppose $d_{(2)}=1$. According to (3i) we get $t^{L}(K G) \geq 14>10$.

Finally, let $p \geq 7$. Since $p^{n-1}>6, \quad p^{n}-4 p+5>p^{n-1}+2 p-1$, so by (iii) of Lemma 1 it follows that this case is impossible. 
Lemma 3. Let $K$ be a field with $\operatorname{char}(K)=p \geq 3$ and $G$ a nilpotent group, such that $\left|G^{\prime}\right|=p^{n}$ and $t^{L}(K G)=p^{n}-4 p+5$. Then $p=5, c l(G)=2$ and $G^{\prime} \cong C_{5} \times C_{5}$.

Proof. Let $\operatorname{char}(K)=p \geq 3$ and $t^{L}(K G)=p^{n}-4 p+5$. Clearly, either (iii) or (iv) of Lemma 2 holds, so we consider the following cases:

Case 1. Let $p=n=3$ and $d_{(2)}=d_{(4)}=d_{(6)}=1$. If $\operatorname{cl}(G)=2$ by Theorem 3.2 of [3] we have that $t^{L}(K G)=t\left(G^{\prime}\right)+1 \leq 12<20$. So assume that $\operatorname{cl}(G) \geq 3$. If $\exp \left(G^{\prime}\right)=3$ we obtain that $\mathfrak{D}_{(3)}(G)=\gamma_{3}(G)$ and $\mathfrak{D}_{(4)}(G)=\gamma_{4}(G)$, so $\gamma_{3}(G)=\langle 1\rangle$, a contradiction.

Now let $\exp \left(G^{\prime}\right)=3^{2}$. If $\operatorname{cl}(G)=3$ then $\mathfrak{D}_{(2)}(G)=\gamma_{2}(G) \cong C_{9} \times C_{3}$ and $\mathfrak{D}_{(3)}(G)=\gamma_{3}(G) \cdot \gamma_{2}(G)^{3}=\left\{C_{9}, C_{3} \times C_{3}\right\}$.

If $\mathfrak{D}_{(3)}(G)$ is not cyclic we have that $\mathfrak{D}_{(4)}(G)=\left(\mathfrak{D}_{(3)}(G), G\right)=\mathfrak{D}_{(3)}(G)$ which is impossible. In the other case, we obtain that

$$
\mathfrak{D}_{(4)}(G)=\left(\mathfrak{D}_{(3)}(G), G\right) \cdot \mathfrak{D}_{(3)}(G)^{3}=\mathfrak{D}_{(3)}(G)
$$

and again it holds that $\left(\mathfrak{D}_{(3)}(G), G\right)=\mathfrak{D}_{(3)}(G)$.

Finally suppose that $\operatorname{cl}(G)=4$. Clearly $\mathfrak{D}_{(3)}(G)=\gamma_{3}(G)$ and

$$
\mathfrak{D}_{(4)}(G)=\left(\mathfrak{D}_{(3)}(G), G\right) \cdot \gamma_{2}(G)^{3}=\gamma_{4}(G) \cdot \gamma_{2}(G)^{3}=\gamma_{3}(G),
$$

so $\gamma_{2}(G)^{3} \subseteq \gamma_{3}(G)$ and by Theorem III.2.13 ([10], p.266), we have that $\gamma_{3}(G)^{3} \subseteq$ $\gamma_{4}(G)$. Now $\mathfrak{D}_{(5)}(G)=\gamma_{4}(G) \cdot \gamma_{3}(G)^{3}=\gamma_{4}(G)$ and

$$
\mathfrak{D}_{(6)}(G)=\gamma_{5}(G) \cdot \gamma_{3}(G)^{3}=\gamma_{4}(G),
$$

so $\gamma_{3}(G) \cong C_{9}$ and $\mathfrak{D}_{(7)}(G)=\gamma_{3}(G)^{3}=\mathfrak{D}_{(6)}(G)$, which is a contradiction.

Case 2. Let $p=5, n=2$ and $d_{(2)}=2$. Obviously, $\gamma_{2}(G) \cong C_{5} \times C_{5}$. If $\operatorname{cl}(G)=3$, then $\mathfrak{D}_{(3)}(G)=\gamma_{3}(G) \neq\langle 1\rangle$, a contradiction.

Proof of the Theorem 1 Follows from Lemmas 2 3 . The equality $t_{L}(K G)=$ $t^{L}(K G)$ is a consequence of (ii) of Theorem 3.2 of 3 .

Lemma 4. Let $K$ be a field with $\operatorname{char}(K)=p>0$ and $G$ a nilpotent group such that $\left|G^{\prime}\right|=p^{n}$. Then $t^{L}(K G)=\left|G^{\prime}\right|-3 p+4$ if and only if one of the following condition holds:

(i) $p=2, \quad n=3, \quad d_{(2)}=2 \quad$ and $\quad d_{(3)}=1$;

(ii) $p=3, \quad n=3 \quad$ and $\quad d_{(2)}=d_{(4)}=d_{(7)}=1$;

(iii) $p=5, \quad n=2 \quad$ and $\quad d_{(2)}=d_{(3)}=1$.

Proof. As in Lemma 2 we can assume that either $p=2$ and $n \geq 3$ or $n \geq 2$.

Let $p=2, n=3$ and suppose that $d_{(2)}=1$. By (3) we have that $t^{L}(K G) \geq 7>$ 6 , so only possible case is $d_{(2)}=2$ and by (i) of Lemma 1 the statement is holds.

Let $n \geq 4$. Using the same arguments as in the proof of the Lemma 2 we obtain that $d_{\left(2^{i}+1\right)}=d_{\left(\left(2^{n-1}-3\right)+1\right)}=1$ and $d_{(j)}=0$, where $0 \leq i \leq n-2, \quad j \neq 2^{i}+1$, $j \neq 2^{n-1}-2$ and $j>1$. The subgroup $H=\mathfrak{D}_{\left(2^{n-1}-2\right)}(G)$ is central of order 2 and from (2) it yields

$$
\begin{aligned}
\mathfrak{D}_{(m+1)}(G) / H & =\prod_{(j-1) 2^{i} \geq m} \gamma_{j}(G)^{2^{i}} / H \\
& =\prod_{(j-1) 2^{i} \geq m} \gamma_{j}(G / H)^{2^{i}}=\mathfrak{D}_{(m+1)}(G / H) .
\end{aligned}
$$


Put $2^{\bar{d}_{(k)}}=\left[\mathfrak{D}_{(k)}(G / H): \mathfrak{D}_{(k+1)}(G / H)\right]$ for $k \geq 1$. It is easy to check that $\bar{d}_{\left(2^{i}+1\right)}=1$ and $\bar{d}_{(j)}=0$, where $0 \leq i \leq n-2, \quad j \neq 2^{i}+1$ and $j>1$.

Clearly, $\left|\gamma_{2}(G / H)\right|=2^{n-1}$ and $t^{L}(K[G / H])=\left|\gamma_{2}(G / H)\right|+1$. So by Lemma 3 of [6] and by Theorem 1 of [6] the group $\gamma_{2}(G / H)$ is either a cyclic 2-group or $C_{2} \times C_{2}$. If $\gamma_{2}(G / H)$ is a cyclic 2-group, then by (a) of Lemma III.7.1 ([10, p.300) we have that $\gamma_{2}(G)$ is abelian, so it is isomorphic to either $C_{2^{n-1}} \times C_{2}$ or $C_{2^{n}}$. If $\gamma_{2}(G)$ is cyclic, then by Theorem 1 of [6] we get $t^{L}(K G)=\left|\gamma_{2}(G)\right|+1$, so we do not consider this case. On the other hand, if $\gamma_{2}(G / H) \cong C_{2} \times C_{2}$, then $\left|\gamma_{2}(G)\right|=8$. Since there are no nilpotent groups with nonabelian commutator subgroup of order 8 (see [7]), we can put that $\gamma_{2}(G)=\left\langle a, b \mid a^{2^{n-1}}=b^{2}=1\right\rangle \cong C_{2^{n-1}} \times C_{2}$.

According to (1) it holds

$$
\mathfrak{D}_{(2)}(G)=\gamma_{2}(G), \quad \mathfrak{D}_{(3)}(G)=\gamma_{3}(G) \cdot\left\langle a^{2}\right\rangle .
$$

Since $\left|\mathfrak{D}_{(2)}(G) / \mathfrak{D}_{(3)}(G)\right|=2$ we obtain one of the following cases:

$$
\begin{array}{lll}
\gamma_{3}(G)=\langle a\rangle, & \gamma_{3}(G)=\langle a b\rangle, & \gamma_{3}(G)=\langle b\rangle, \\
\gamma_{3}(G)=\left\langle a^{2^{j}} b\right\rangle, & \gamma_{3}(G)=\left\langle a^{2^{j}}, b\right\rangle, & (1 \leq j \leq n-2) .
\end{array}
$$

We consider each of these cases:

Case 1. Let either $\gamma_{3}(G)=\langle a\rangle$ or $\gamma_{3}(G)=\langle a b\rangle$. Since $\gamma_{2}(G)^{2} \subset \gamma_{3}(G)$, by Theorem III.2.13 ([10], p.266), we have that $\gamma_{k}(G)^{2} \subseteq \gamma_{k+1}(G)$ for every $k \geq 2$. It follows that $\gamma_{2}(G)^{2}=\gamma_{4}(G)$. Moreover, $\gamma_{3}(G)^{2} \subseteq \gamma_{5}(G)$. Indeed, the elements of the form $(x, y)$, where $x \in \gamma_{2}(G)$ and $y \in G$ are generators of $\gamma_{3}(G)$, so we have to prove that $(x, y)^{2} \in \gamma_{5}(G)$. By (5)

$$
\left(x^{2}, y\right)=(x, y)(x, y, x)(x, y)=(x, y)^{2}(x, y, x)^{(x, y)}
$$

and $\left(x^{2}, y\right),(x, y, x)^{(x, y)} \in \gamma_{5}(G)$, so $(x, y)^{2} \in \gamma_{5}(G)$ and $\gamma_{3}(G)^{2} \subseteq \gamma_{5}(G)$. Thus $\left|\gamma_{3}(G)\right|=2$, a contradiction.

Case 2. Let $\gamma_{3}(G)=\langle b\rangle$. Now, let us compute the weak complement of $\gamma_{3}(G)$ in $\gamma_{2}(G)$ (see [3], p.34). It is easy to see that, with the notation of [3] (see p.34)

$$
P=\gamma_{2}(G), \quad H=\langle b\rangle, \quad H \backslash P^{2}=\{b\}
$$

so $\nu(b)=2$ and the weak complement is $A=\langle a\rangle$. Since $G$ is of class 3 , by (ii) of Theorem 3.3 ([3], p.43) we have

$$
\begin{aligned}
t_{L}(K G)=t^{L}(K G) & =t\left(\gamma_{2}(G)\right)+t\left(\gamma_{2}(G) /\langle a\rangle\right) \\
& =2^{n-1}+3 \neq\left|G^{\prime}\right|-2 .
\end{aligned}
$$

Case 3. Let $\gamma_{3}(G)=\left\langle a^{2^{j}} b\right\rangle$ with $1 \leq j \leq n-2$. Since $d_{(3)}=1$, by (11) we get

$$
\mathfrak{D}_{(2)}(G)=\gamma_{2}(G), \quad \mathfrak{D}_{(3)}(G)=\left\langle a^{2}, b\right\rangle, \quad \mathfrak{D}_{(4)}(G)=\left(\left\langle a^{2}, b\right\rangle, G\right) \cdot\left\langle a^{4}\right\rangle,
$$

and $\mathfrak{D}_{(4)}(G)$ is one of the following groups: $\left\langle a^{2}\right\rangle,\left\langle a^{2} b\right\rangle,\left\langle a^{4}, b\right\rangle$.

Suppose that $\mathfrak{D}_{(4)}(G)=\left\langle a^{2}\right\rangle$. Since $d_{(4)}=0$ we have

$$
\mathfrak{D}_{(5)}(G)=\left(\left\langle a^{2}\right\rangle, G\right) \cdot \mathfrak{D}_{(3)}(G)^{2}=\left(\left\langle a^{2}\right\rangle, G\right) \cdot\left\langle a^{4}\right\rangle=\left\langle a^{2}\right\rangle .
$$

The last equality forces $\left(\left\langle a^{2}\right\rangle, G\right)=\left\langle a^{2}\right\rangle$ and so $\mathfrak{D}_{(k)}(G)=\left\langle a^{2}\right\rangle$ for each $k \geq 5$, which is impossible.

Now let $\mathfrak{D}_{(4)}(G)=\left\langle a^{2} b\right\rangle$. Similarly, because $d_{(4)}=0$, we get

$$
\mathfrak{D}_{(4)}(G)=\mathfrak{D}_{(5)}(G)=\left(\left\langle a^{2} b\right\rangle, G\right) \cdot\left\langle a^{4}\right\rangle=\left\langle a^{2} b\right\rangle
$$

and $\left(\left\langle a^{2} b\right\rangle, G\right)=\left\langle a^{2} b\right\rangle$, which is impossible. 
Finally, suppose that $\mathfrak{D}_{(4)}(G)=\left\langle a^{4}\right\rangle \times\langle b\rangle$ and exists $k \leq 2^{n-2}+1$, such that $\mathfrak{D}_{(k)}(G)$ is cyclic. Using the same arguments of above, we obtain that $\mathfrak{D}_{(m)}(G) \neq$ $\langle 1\rangle$ for each $m$, which is impossible.

Therefore $\mathfrak{D}_{\left(2^{n-2}+1\right)}(G)=\left\langle a^{2^{n-2}}\right\rangle \times\langle b\rangle$. Since $d_{\left(2^{n-2}+1\right)}=1$ and $d_{\left(2^{n-2}+2\right)}=0$, by (11) we get

$$
\begin{aligned}
\mathfrak{D}_{\left(2^{n-2}+2\right)}(G) & =\left(\mathfrak{D}_{\left(2^{n-2}+1\right)}(G), G\right) \cdot \mathfrak{D}_{\left(2^{n-3}+2\right)}(G)^{2} \\
& =\left(\mathfrak{D}_{\left(2^{n-2}+1\right)}(G), G\right)=\left\langle\omega \mid \omega^{2}=1\right\rangle ; \\
\mathfrak{D}_{\left(2^{n-2}+3\right)}(G) & =\left(\mathfrak{D}_{\left(2^{n-2}+2\right)}(G), G\right)=(\langle\omega\rangle, G)=\langle\omega\rangle .
\end{aligned}
$$

It follows that $(\langle\omega\rangle, G)=\langle\omega\rangle$ and $\mathfrak{D}_{(k)}(G)=\langle\omega\rangle$ for all $k \geq 2^{n-2}+2$, again a contradiction.

Case 4. Let $\gamma_{3}(G)=\left\langle a^{2^{j}}\right\rangle \times\langle b\rangle$ with $1 \leq j \leq n-2$. It is easy to check that this case is similar to the last subcase of the previous case.

Therefore the case $p=2$ is finished.

Let now $p=3$ and $n \geq 4$. Using the same arguments as in the previous case, we get $d_{\left(3^{i}+1\right)}=d_{\left(\left(3^{n-1}-3\right)+1\right)}=1$ for $0 \leq i \leq n-2$. Since $\exp \left(G^{\prime}\right) \leq 3^{n-1}$ and $3^{n-2} \mid 2 \cdot 3^{n-2}$, by (ii) of Lemma 1 it yields that $\mathfrak{D}_{\left(2 \cdot 3^{n-2}+1\right)}(G)=\langle 1\rangle$, so $d_{\left(\left(3^{n-1}-3\right)+1\right)}=0$, a contradiction.

Let $p=3$ and $n=3$. Similarly to prof of part (ii) of Lemma 2, we obtain that $d_{(2)}=d_{(4)}=d_{(7)}=1$, so this case is complete.

For $p=5$, the part (iii) of Lemma 1 gives only one possible case: $n=2$, which follows that $d_{(2)}=1$ and $d_{(3)}=1$.

Lemma 5. Let $K$ be a field with char $(K)=2$ and $G$ a nilpotent group such that $\left|G^{\prime}\right|=2^{n}$. If $t^{L}(K G)=2^{n}-2$, then either (i) or (ii) of Theorem 2 hold.

Proof. By (i) of Lemma 4 we have $n=3, \quad d_{(2)}=2$ and $d_{(3)}=1$. If $\gamma_{2}(G)$ is elementary abelian, according to Theorem 1 and Theorem 1 of [5] we have that $\operatorname{cl}(G)=3$. If $\left|\gamma_{3}(G)\right|=4$, then it is easy to check $d_{(2)}=1$, a contradiction. Therefore $\gamma_{2}(G) \cong C_{4} \times C_{2}$ and the statement follows at once by easy calculation.

Lemma 6. Let $K$ be a field with $\operatorname{char}(K)=p \geq 3$ and $G$ a nilpotent group, such that $\left|G^{\prime}\right|=p^{n}$. If $t^{L}(K G)=p^{n}-3 p+4$ then $p=5, \operatorname{cl}(G)=3$ and $\gamma_{2}(G) \cong C_{5} \times C_{5}$.

Proof. Using the same argument of proof of Lemma 3 , we obtain that $p \neq 3$. If $p=5$ and $n=2$, then $\quad d_{(2)}=d_{(3)}=1$ and it follows that $\gamma_{2}(G)$ is not central, so the proof is complete.

Proof of the Theorem 2. Follows from Lemma 5, Lemma 6 and Theorem 1. The equality $t_{L}(K G)=t^{L}(K G)$ is an immediate consequence of part (ii) of Theorem 3.2 by [3] and part (ii) of Theorem 3.3 by [3].

Lemma 7. Let $K$ be a field with $\operatorname{char}(K)=p>0$ and $G$ a nilpotent group such that $\left|G^{\prime}\right|=p^{n}$. Then $t^{L}(K G)=\left|G^{\prime}\right|-2 p+3$ if and only if one of the following condition holds:

(i) $p=2, \quad n=3, \quad d_{(2)}=1 \quad$ and $\quad d_{(3)}=2$;

(ii) $p=3, \quad n=2 \quad$ and $\quad d_{(2)}=2$. 
Proof. Let $t^{L}(K G)=\left|G^{\prime}\right|-2 p+3$. By (iii) of Lemma 1 we can assume that $p \leq 3$.

Let $p=2$ and $n=3$. According to (i) of Lemma 2, (i) of Lemma 4 and (3) it is easy to check that $d_{(2)}=1$ and $d_{(3)}=2$.

If $n \geq 4$, then using the same technic as in the proof of Lemma 2 and Lemma 7 we obtain that $\quad d_{\left(2^{i}+1\right)}=d_{\left(2^{n-1}-1\right)}=1 \quad$ and $\quad d_{(j)}=0$, where $0 \leq i \leq n-2$, $j \neq 2^{i}+1, \quad j \neq 2^{n-1}-1 \quad$ and $j>1$;

Put $m=2^{n-2}-1$ and $l=2 m$. Since $d_{\left(\left(2^{n-2}-1\right)+1\right)}=0 \quad$ and $\quad \nu_{2^{\prime}}(l)=\nu_{2^{\prime}}(m)$, by (v) of Lemma 1 we get $d_{\left(\left(2^{n-1}-2\right)+1\right)}=0$, a contradiction.

Finally, let $p=3$ and $n \geq 3$. In this case as in proof of (ii) of Lemma 2, we obtain the contradiction and the proof is complete.

Lemma 8. Let $K$ be a field with $\operatorname{char}(K)=2$ and $G$ a nilpotent group such that $\left|G^{\prime}\right|=2^{n}$. If $t^{L}(K G)=2^{n}-1$, then either (i) or (ii) of Theorem 3 holds.

Proof. If $n \geq 4$, then the proof is the same of that used in Lemma 3 for $n \geq 5$. So assume that $n=3$.

Therefore either $\gamma_{2}(G) \cong C_{2} \times C_{2} \times C_{2}$ or $\gamma_{2}(G) \cong C_{4} \times C_{2}$. In the first case, part (i) of Theorem [1 and part (i) of Theorem 2 force that $\gamma_{3}(G)$ to be of order 4 and central. In the last one since $\left|\mathfrak{D}_{(2)}(G) / \mathfrak{D}_{(3)}(G)\right|=2$ and $\mathfrak{D}_{(3)}(G)=$ $\gamma_{3}(G) \cdot \gamma_{2}(G)^{2}$ we obtain that $\gamma_{3}(G)$ is one of the following groups:

$$
\begin{gathered}
\gamma_{3}(G)=\langle a\rangle, \quad \gamma_{3}(G)=\langle a b\rangle, \quad \gamma_{3}(G)=\langle b\rangle, \\
\gamma_{3}(G)=\left\langle a^{2} b\right\rangle, \quad \gamma_{3}(G)=\left\langle a^{2}, b\right\rangle .
\end{gathered}
$$

If $\gamma_{3}(G)=\langle a\rangle$ or $\gamma_{3}(G)=\langle a b\rangle$ the proof was made in Case 1 of Lemma 3 .

Let either $\gamma_{3}(G)=\langle b\rangle$ or $\gamma_{3}(G)=\left\langle a^{2} b\right\rangle$. It is easy to check that in both cases the week complement of $\gamma_{3}(G)$ in $\gamma_{2}(G)$ (see [3], p.34) is $A=\langle a\rangle$ and $t^{L}(K G)=$ $t_{L}(K G)=7$.

Finally, let $\gamma_{3}(G)$ be noncyclic. Obviously, $\mathfrak{D}_{(3)}(G)=\gamma_{3}(G)$ and $\gamma_{3}(G)^{2}=\langle 1\rangle$, so $\mathfrak{D}_{(4)}(G)=\langle 1\rangle$ and the statement holds.

Proof of the Theorem 3. Let $K$ be a field with $\operatorname{char}(K)=p \geq 3$. By Lemma 7 it holds that $\gamma_{2}(G)$ is noncyclic of order 9 . If $p=2$ the statement was proved in Lemma 8

Proof of the Theorem 4. Let $p=2$ and $t_{L}(K G)=\left|G^{\prime}\right|-2 p+3$. Note that in [4, 6, 5] we obtained the description of Lie nilpotent group algebras $K G$ with $t^{L}(K G) \in\left\{\left|G^{\prime}\right|-p+2, \quad\left|G^{\prime}\right|+1\right\}$. Since $t_{L}(K G) \leq t^{L}(K G)$ and $\quad\left|G^{\prime}\right|-2 p+3<$ $\left|G^{\prime}\right|-p+2<\left|G^{\prime}\right|+1, \quad$ we have that

$$
t^{L}(K G) \in\left\{\left|G^{\prime}\right|-2 p+3, \quad\left|G^{\prime}\right|-p+2, \quad\left|G^{\prime}\right|+1\right\} .
$$

If $t^{L}(K G) \in\left\{\left|G^{\prime}\right|-p+2, \quad\left|G^{\prime}\right|+1\right\}$, then according to [4, 6, 5], respectively, we have that $t_{L}(K G) \in\left\{\left|G^{\prime}\right|-p+2, \quad\left|G^{\prime}\right|+1\right\}$, a contradiction. Therefore $t^{L}(K G)=\left|G^{\prime}\right|-2 p+3$ and by Theorem 3 we get $t_{L}(K G)=t^{L}(K G)$ and the description of groups $G$ is done.

Let $p=3$. First, by (3) there is no $K G$ with $t^{L}(K G)=\left|G^{\prime}\right|-2 s$ with $s \in \mathbb{N}$, because $t^{L}(K G)=2+2\left(\sum_{q \geq 1} q d_{q+1}\right) \neq 3^{n}-2 s$. Using this remark, the proof is the same as for case $p=2$.

Now the proof of the parts (i) and (ii) of the Theorem 4 for $p=2$ and $p=3$ is the same. Note that for $p \geq 5$, the proof is a trivial consequence of the Bhandari-Passi's Theorem [1]. 


\section{REFERENCES}

[1] A. K. Bhandari and I. B. S. Passi. Lie-nilpotency indices of group algebras. Bull. London Math. Soc., 24(1):68-70, 1992.

[2] A. A. Bovdi and I. I. Khripta. Generalized Lie nilpotent group rings. Mat. Sb. (N.S.), 129(171)(1):154-158, 160, 1986.

[3] A. A. Bovdi and J. Kurdics. Lie properties of the group algebra and the nilpotency class of the group of units. J. Algebra, 212(1):28-64, 1999.

[4] V. Bovdi. Modular group algebras with almost maximal lie nilpotency indices II. Sci. Math. Jpn., to appear:1215-1219, 2006.

[5] V. Bovdi, T. Juhász, and E. Spinelli. Modular group algebras with almost maximal Lie nilpotency indices. Algebr. Represent. Theory, 9(3):259-266, 2006.

[6] V. Bovdi and E. Spinelli. Modular group algebras with maximal Lie nilpotency indices. Publ. Math. Debrecen, 65(1-2):243-252, 2004.

[7] W. Burnside. On some properties of groups whose orders are power of primes. Proc. London Math. Soc., 11:225-245, 1913.

[8] X. K. Du. The centers of a radical ring. Canad. Math. Bull., 35(2):174-179, 1992.

[9] N. Gupta and F. Levin. On the Lie ideals of a ring. J. Algebra, 81(1):225-231, 1983.

[10] B. Huppert. Endliche Gruppen. I. Die Grundlehren der Mathematischen Wissenschaften, Band 134. Springer-Verlag, Berlin, 1967.

[11] I. I. Khripta. The nilpotence of the multiplicative group of a group ring. Mat. Zametki, 11:191-200, 1972.

[12] I. B. S. Passi. Group rings and their augmentation ideals, volume 715 of Lecture Notes in Mathematics. Springer, Berlin, 1979.

[13] I. B. S. Passi, D. S. Passman, and S. K. Sehgal. Lie solvable group rings. Canad. J. Math., 25:748-757, 1973.

[14] A. Shalev. Applications of dimension and Lie dimension subgroups to modular group algebras. Ring theory 1989, Ramat Gan and Jerusalem, 1988/1989, Israel Math. Conf. Proc., 1:84-95, 1989.

[15] A. Shalev. Lie dimension subgroups, Lie nilpotency indices, and the exponent of the group of normalized units. J. London Math. Soc. (2), 43(1):23-36, 1991.

[16] A. Shalev. The nilpotency class of the unit group of a modular group algebra. III. Arch. Math. (Basel), 60(2):136-145, 1993.

[17] R. K. Sharma and V. Bist. A note on Lie nilpotent group rings. Bull. Austral. Math. Soc., 45(3):503-506, 1992

V. Bovdi, Institute of Mathematics, University of Debrecen, H-4010 Debrecen, P.O.Box 12, Hungary

Institute of Mathematics and Informatics, College of Nyíregyháza, Sóstói út 31/B,

H-4410 NYÍREgYhÁzA, Hungary

E-mail address: vbovdi@math.klte.hu

J. B. Srivastava, Department of Mathematics, Indian Institute of Technology, Delhi, New Delhi-110016, India

E-mail address: jbsrivas@gmail.com 